\title{
Gall bladder carcinoma: the facts and the mimics
}

\author{
Niharika Prasad ${ }^{1,2^{*}}$ and Saugata $\operatorname{Sen}^{3}$
}

\begin{abstract}
Background: Gall bladder carcinoma (GBC) is the most common biliary epithelial neoplasm. Its slow progression and late presentation lend it a poor prognosis. The risk factors can be divided into cholelithiasis, inflammatory causes, infection, exposure, and gall bladder pathologies. It usually spreads by hematogenous or lymphatic route or directly invades the liver. There are many controversies related to guidelines for management of gall bladder polyps and treatment options.

Main text: This review article attempts to give definitive guidelines for the same and helps the reader distinguish it from other benign mimickers. The emerging role of newer modalities like contrast ultrasound, elastography, and magnetic resonance cholangiopancreatography has also been briefly mentioned. This paper reviews the literature to provide concise background, etiopathogenesis, radiological findings, and management options of GBC.

Conclusions: Out of all the available modalities MDCT, FDG-PET CT, and image-guided biopsies play the most important role in diagnosis and follow-up. Imaging remains elementary in pre-operative planning and management of gall bladder neoplasms.
\end{abstract}

\section{Background}

Gall bladder carcinoma is the fifth most common gastro-intestinal neoplasm and the most common biliary tract malignancy in the world [1]. The purpose of this review article is to highlight the imaging findings which can aid in the early diagnosis and differentiation from other pathologies. Since the clinical symptoms can be non-specific, the diagnosis is often late, resulting in a poor prognosis. The spread of gall bladder carcinoma $(\mathrm{GBC})$ to the liver parenchyma and the adjacent internal organs is due to lack of serosa in gall bladder wall, proximity, cholecystic veins draining into liver portal vein, lymphatics from GB draining into the liver [2] (Fig. 1). This article will focus on the role of cross-sectional modalities, imaging findings with advances in imaging mimics, staging, and new treatment approaches. Although

\footnotetext{
* Correspondence: vats.niharika248@gmail.com

'Department of Radiology, Dr.D.Y.Patil Medical College, Hospital \& Research Centre, Room No. A-42, Old Staff Quarter, Dr.D.Y.Patil Hospital, Pimpri, Pune, Maharashtra 411018, India

${ }^{2}$ All India Institute of Medical Sciences, Patna, Bihar, India

Full list of author information is available at the end of the article
}

there are several review articles on foreign data regarding epidemiology and imaging of gall bladder (GB) malignancy, very few recent systematic reviews are available, especially regarding endemic Indian states.

According to AJCC (American Joint Committee on Cancer), primary GB carcinoma has been classified as $\mathrm{T} 1$, confined to lamina propria or the muscle layer of the $\mathrm{GB}$ (T1A and T2B, respectively), T2 extending to serosa, T3 perforating the serosa or directly invading the adjacent structure, T4 invading the main portalvein, hepatic artery or distant organs (Table 1). Lesion extends through the muscle layer into the fibrous tissue on the side of the peritoneum in T2a stage. In T2b stage, lesion extends through the muscle layer into the fibrous tissue on the side of the liver. The most important prognostic factor for predicting survival is nodal status; effective lymph node dissection is thus valuable [3]. Lymph nodal spread occurs in a predictable manner which is due to the drainage pattern. Initially, cystic duct and pericholedochal nodes are involved, followed by distant metastasis to nodes adjacent to the head of pancreas and then to aortocaval nodes. Low sensitivities 


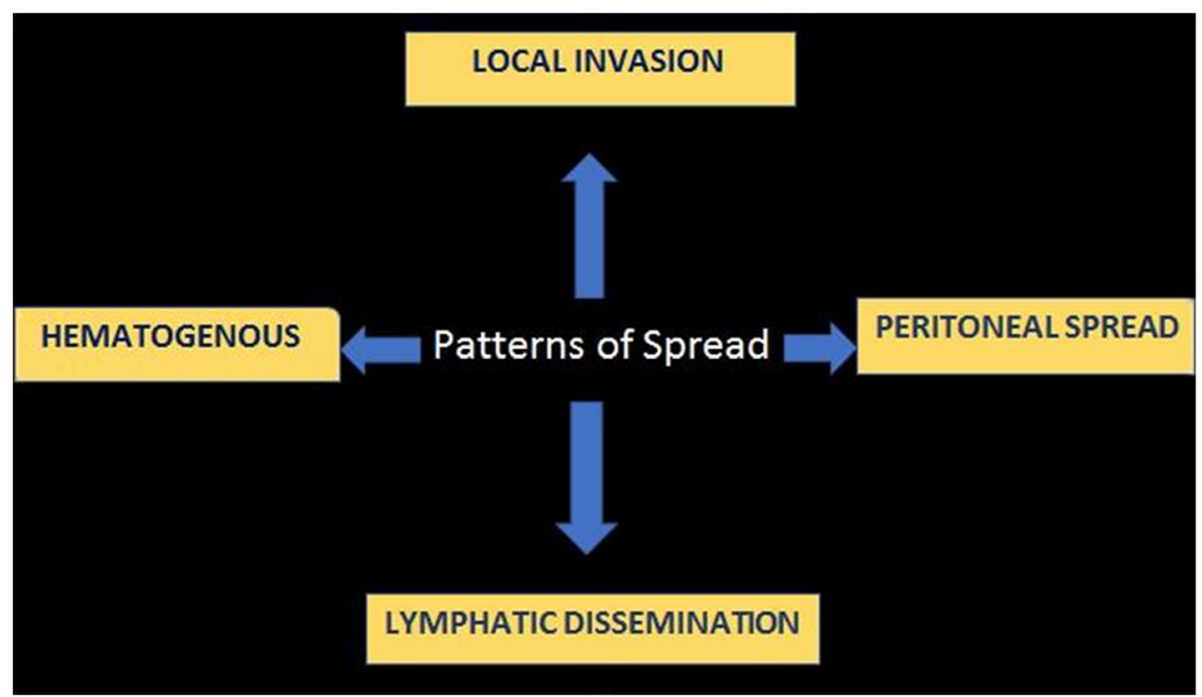

Fig. 1 Patterns of spread of gall bladder carcinoma

have been reported for the detection of positive nodes on CECT abdomen. However, involvement of N1 or N2 nodal stations is not a criterion for non-resectability of GB carcinoma [4]. Intraoperative perforation of gall bladder carries a risk of local recurrence and bile spillage can cause peritoneal carcinomatosis [5]. When a GB neoplasm is suspected preoperatively, it is contraindicated to do a laparoscopic cholecystectomy.

An interesting study conducted in Tata Memorial Hospital, Mumbai, India, stated that one in every two patients who presented to a tertiary cancer center with a high index of suspicion for malignancy based on clinicoradiological findings proved benign on final histology. This highlights the fact that, for radiologically suspected gall bladder malignancy, we need to have a confirmed histological diagnosis at least during surgery before proceeding to radical resection. An intraoperative frozen section can be used to confirm the same. Radiological diagnosis alone risks overtreatment of benign disease in suspected gall bladder cancer [6].

\section{Methods}

A PubMed search using keywords 'gall bladder carcinoma (or) cancer (or) neoplasm,' 'differentials,' 'advances in treatment,' 'incidental,' 'pathology,' 'surgery,' and 'review article' was carried out, and articles published in last 10 years from present were included. Other biliary tract malignancies were excluded. Studies focusing on Asian/Indian subcontinent and high incidence countries were given priority.

\section{Epidemiology and pathogenesis}

The incidence of GBC in the USA is three per one lakh population. Well- to moderately differentiated subtypes are more common. It is three times more common in females than in males and more common above 65 years of age [1]. Regions with high prevalence include India (New Delhi, Bihar, U.P, and Bhopal), La Paz, Bolivia, Pakistan, and Ecuador with high rates reported in Chile, Poland, Japan, and Israel [3]. According to the Indian Council of Medical Research (ICMR) (1990-1996), there is a 10 times lower incidence of GBC per 100,000 in South India compared with the North, the age-adjusted incidence rate for females being 0.8 in Chennai in the South and 8.9 in Delhi in the North. GBC ranks among the first 10 cancers in the ICMR registries (2006-2008) of Delhi, Dibrugarh, Kolkata, Bhopal, and Mumbai [7].

According to few authors from Banaras Hindu University, India, Eastern UP, and Western Bihar, the Gangetic belt has the highest incidence of GBC. They attribute this to environmental factors, water pollutants, industrial, and agricultural effluents containing aromatic hydrocarbons, nitrosamines, and chemicals such as nitrates and nitrites which are by-products from domestic sewage [8]. Typhoid infection is prevalent in this region which may also be associated with the gall bladder carcinogenesis. Adulteration in cooking oil (mustard) by sanguinarine and diethylnitrosamine has also been found to be linked with malignancy. They thus conclude that GBC has multifactorial etiology [8].

The risk factors can be divided into cholelithiasis, inflammatory causes, infection, exposure, and gall bladder pathologies or abnormalities. Seventy to $90 \%$ of GBC patients have cholelithiasis and it is presumed that chronic mucosal irritation by calculi leads to dysplasia [3] (Tables 2 and 3). Approximately 25\% of the cases of porcelain gall bladder (which is one of the last stages of chronic inflammation) are associated with GBC [3]. 
Table 1 AJCC stage AJCC staging system

\begin{tabular}{lll}
\hline $\begin{array}{l}\text { AJCC } \\
\text { stage }\end{array}$ & $\begin{array}{l}\text { Stage } \\
\text { group }\end{array}$ & Description \\
\hline 0 & Tis & $\begin{array}{l}\text { Lesion is only in the epithelium and has not } \\
\text { grown into deeper layers of the gall bladder (Tis) }\end{array}$
\end{tabular}

NO

$\mathrm{MO}$

I T1

NO

MO

$\|$ T2a

NO

MO

$\| \mathrm{B} \quad \mathrm{T} 2 \mathrm{~b}$

NO

MO

IIIA

$$
\mathrm{T} 1-3
$$

NO

$\mathrm{MO}$

IVA T4

T4 The tumor has grown into portal vein or hepatic artery or it has grown into 2 or more structures outside of the liver (T4). It may or may not have spread to no more than 3 nearby lymph nodes (N0 or N1).

N0 or N1

\section{Mo No distant spread.}

IVB Any $T$

The primary tumor may or may not have infiltrated out of the gall bladder. Spread to 4 or more regional lymph nodes (N2).

N2

$\mathrm{MO}$

$\mathrm{OR}$

Any $T \quad$ It has spread to distant sites such as the liver, peritoneum, or the lungs (M1).

Any $\mathrm{N}$

M1

Clinical presentation includes abdominal pain, weight loss, fever, and jaundice. It may however be detected incidentally, ranging from 0.2 to $2 \%$ in incidence [9].
Gall bladder polyps which are at higher risk of malignancy are $>10 \mathrm{~mm}$ and sessile types while pseudo polyps and cholesterol polyps carry less risk. Overall, any polyp > $10 \mathrm{~mm}$ in size, patient age $>60$ years, interval increase in size and presence of gall stones are considered criteria for prophylactic removal [1] (Table 4).

\section{Genetic mutations and molecular pathogenesis}

Two independent biological pathways have been described: (a) dysplasia-carcinoma sequence (more common) and (b) an adenoma-carcinoma sequence. Kras and TP53 are the most extensively researched genes implicated in gall bladder cancer. Carcinogenic pathways may include (i) inflammation secondary to gallstones leading to p53 mutations and eventual carcinoma, (ii) point mutation of Kras contributing to hyperplasia then carcinoma as seen in patients with an anomalous junction of pancreaticobiliary duct, and (iii) neoplastic areas in gall bladder polyps secondary to Kras mutation [3].

\section{Pathological types of GBC}

In a study conducted by a few pathologists from AIIMS, New Delhi, India, the authors attempted to study the role of guided fine needle aspiration cytology (FNAC) for diagnosis of GBC and to evaluate the feasibility of applying world health organization (WHO) classification on fine needle aspiration sample to predict the outcomes [10].

Adenocarcinoma was the most frequent diagnosis in their study (86.7\% of cases) and all well-differentiated carcinomas were papillary carcinomas. WHO classification (2010) divides GBC into various morphologic subtypes with their associated prognostic outcomes. It classifies conventional adenocarcinoma into intestinal, gastric foveolar and biliary subtypes in addition to mucinous, signet cell, clear cell, and hepatoid variants. Uncommon variants carrying poor prognosis such as mucinous carcinoma, squamous cell carcinoma, small cell carcinoma, and undifferentiated carcinoma can be diagnosed on the aspirated material.

\section{Imaging characteristics}

Gall bladder carcinoma can present in the following ways: (1) intraluminal mass lesion, (2) focal/diffuse

Table 2 Risk factor and percentage of contribution

\begin{tabular}{ll}
\hline Risk factor & Percentage of contribution \\
\hline Cholelithiasis & $70-90 \%$ \\
Porcelain GB & $<1 \%$ \\
GB Polyp & $2-3 \%$ \\
Pancreatico biliary anomalies & $<10 \%$ \\
Exposures & $1 \%$ \\
Infections & $2-5 \%$ \\
\hline
\end{tabular}


Table 3 Demographic factors

\begin{tabular}{lll}
\hline Demographic factors & Gall bladder pathologies/abnormalities & Exposures \\
\hline Advanced age & Cholelithiasis & Heavy metals \\
Female gender & Porcelain gall bladder & Medications:methyldopa, OCP, \\
isoniazid, estrogen \\
Obesity & Gall bladder polyps & Smoking \\
Geography & Congenital biliary cysts & Infections: Salmonella, Helicobacter \\
Ethnicity & Pancreaticobiliary anatomical anomalies & \\
Genetic predisposition & & \\
\hline
\end{tabular}

asymmetric wall thickening, or (3) polyp (Fig. 2). The first imaging tool for the gall bladder is an abdominal ultrasound. An article by few Spanish authors reviewed the most relevant ultrasound findings associated with gall bladder cancer [11]. They found the pattern of mass replacing gall bladder lumen the most common type. Hyperechoic foci and acoustic shadow in the mass may be related to the coexistence of calculi. Direct spread to the area around the liver and the biliary tree is common, and it may be impossible to establish an ultrasound limit between the mass and the liver parenchyma. Differential diagnosis includes metastases, hepatocellular carcinoma, and cholangiocarcinoma.

The next presentation can be focal or diffuse wall thickening. A thickened GB wall measures more than 3 $\mathrm{mm}$. Non-specific GB wall thickening can be due to cirrhosis, dengue, and viral hepatitis. Wall thickening over $12 \mathrm{~mm}$ which is irregular, marked wall asymmetry, loss of interface between the gall bladder wall and the liver, wall calcifications, lymphadenopathy, and bile duct obstructions are more in favor of malignancy.

A third pattern of GBC presentation is polyp and differentiation of a polyp from sludge is possible by changing the patient's position. Benign polyps include cholesterolosis (majority), adenomyosis, inflammatory, and hyperplastic types. As mentioned earlier (under pathogenesis), size and morphology constitute important criteria for decision on management of suspicious polyps. The wall adjacent to the polyp should be normal,

Table 4 Criteria for prophylactic removal

\begin{tabular}{ll}
\hline Reference no. & $\begin{array}{l}\text { Predictive cut-off size for } \\
\text { malignant polyp at which } \\
\text { excision is recommended }\end{array}$ \\
\hline 1 & $>2 \mathrm{~cm}$ \\
2 & $>1 \mathrm{~cm}$ \\
9 & $>1 \mathrm{~cm}$ \\
11 & $>=15 \mathrm{~mm}$ \\
12 & $>1 \mathrm{~cm}$ \\
13 & $>=12 \mathrm{~mm}$ \\
\hline
\end{tabular}

and irregularity or focal thickening $>3 \mathrm{~mm}$ can be a hint towards malignancy [12]. Age more than 40 years was also found to be a finding in resected polyps proved to be adenocarcinoma [13]. Sometimes GB sludge can be confused with GBC, identifying the twinkling artifact which can be produced by sludge and differentiating it from flow on spectral Doppler can be helpful to exclude a gall bladder mass [14].

Cross-sectional imaging features show imaging of gall bladder carcinoma typically demonstrates heterogeneous echo texture within an intraluminal mass or asymmetric wall thickening in early stages. A standard CT protocol in cases where there is a strong suspicion for a biliary tract or gall bladder cancer, arterial phase images can be acquired at 25 to $30 \mathrm{~s}$, followed by venous phase images at 50 to $60 \mathrm{~s}$. In those cases where the gall bladder carcinoma is an incidental finding and there is no prospective suspicion for biliary malignancy, the CT protocol can comprise of only a single-phase study (venous phase images at 50-60 s) [15].

Various wall enhancement patterns have been described as characteristic of different GB pathologies to differentiate benign from malignant causes. Type 1 was a one-layer pattern, and types 2-5 were two-layer patterns. The type 1 pattern was a heterogeneously enhancing one-layer $\mathrm{GB}$ wall or indistinguishable layering of the GB wall; type 2, strongly enhancing thick inner layer and weakly enhancing or non-enhancing outer layer; these are characteristic of malignant types. Type 3 showed borderline enhancement and thickness of the inner layer with small cystic spaces and non-enhancing outer layer; as seen in adenomyomatosis. Type 4, weakly enhancing thin inner layer and non-enhancing thin outer layer; seen in chronic cholecystitis, and type 5, weakly enhancing thin inner layer and non-enhancing thick outer layer as seen in acute cholecystitis and dengue $[16,17]$. The sensitivity and specificity of type 1 enhancement pattern on computed tomography for predicting the GB malignancy according to this study were $90.476 \%$ and $97.43 \%$, respectively. The positive and negative predictive values were found to be $95 \%$ and $95 \%$, respectively [16]. 

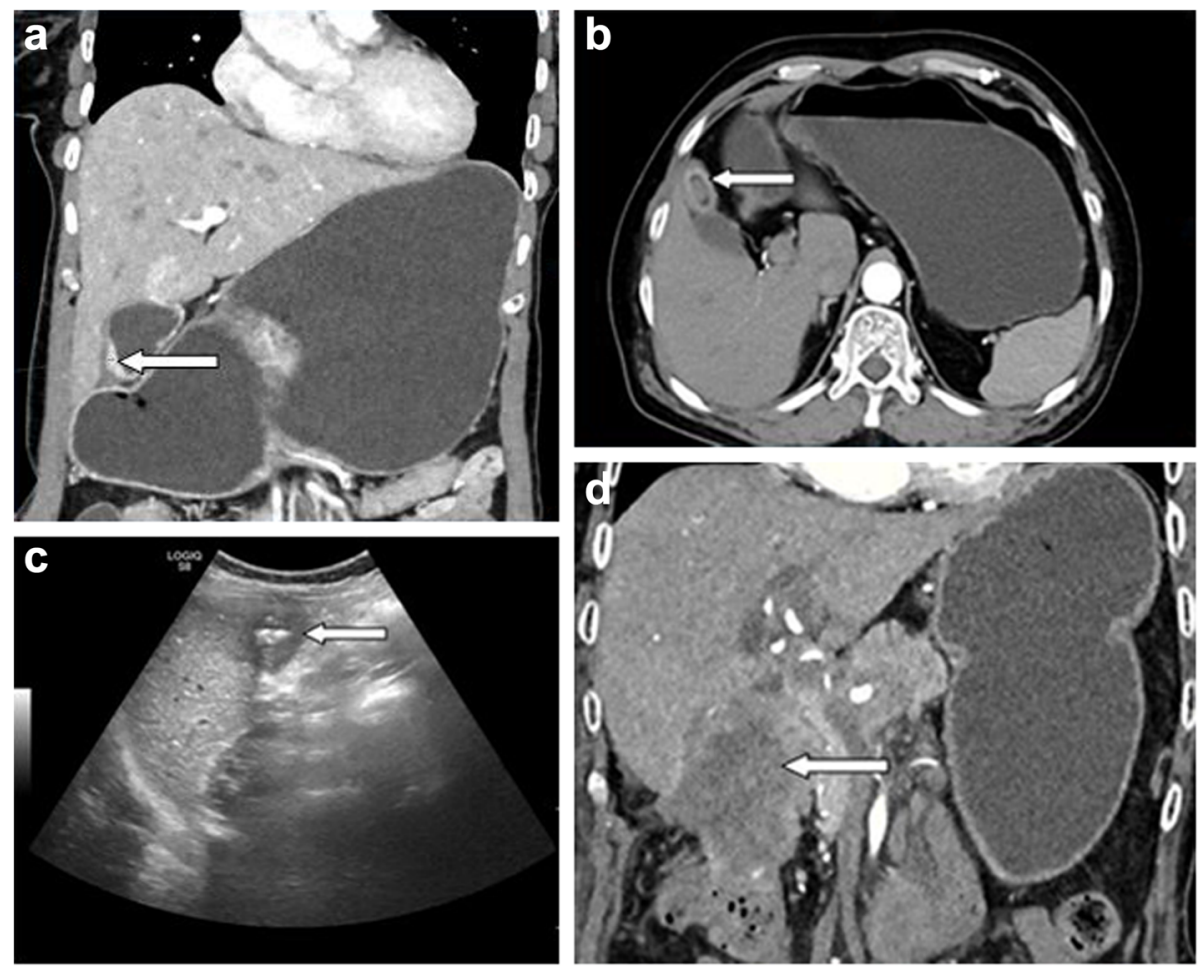

Fig. 2 Different forms of presentation of gall bladder carcinoma (a-d)

In our previously published study in a region of India endemic for CA GB, we have reported the sensitivity and specificity of MDCT in detection of carcinoma of gall bladder to be $92.8 \%$ and $96.7 \%$, respectively [18] (Table 5). We found that, triple-phase MDCT with 3D reconstruction is a comprehensive imaging technique for staging gall bladder carcinoma and determining the vascular road map before surgery. It also aids in identifying anatomical variants pre-operatively.

On non-contrast computed tomography (CT), gall bladder carcinoma is typically hypodense; $40 \%$ demonstrate hyper vascular foci equal to or greater than liver parenchyma on post-contrast. On magnetic resonance (MRI), GB carcinoma has intermediate T1 and moderately hyperintense T2 signal intensity. On both CT and MR, intense irregular enhancement may occur at the periphery of the lesion on arterial phase imaging with persistent portal venous and delayed enhancement [12]. This persistence of enhancement in portal venous and delayed phases can help to differentiate it from large hepatocellular carcinomas which have tendency to wash out $[1,12]$.

Early detection of GB neoplasms can increase by magnifying the porta region to look for sub centimeter sized lymph nodes and fat stranding. Reformatted multiplanar images are helpful to look for local invasion like common bile duct and vascular infiltration.

The combination of MRI with MRA (magnetic resonance angiography) and MRCP (magnetic resonance cholangiopancreatography) is useful in detecting vascular invasion and biliary tract involvement. The main advantage of MRI over contrast-enhanced $\mathrm{CT}$ is its ability to demonstrate liver secondaries better $[1,3,12]$. The addition of diffusion weighted images and ADC (apparent diffusion coefficient) cut-off values has not been stressed in most studies. An ideal MRI protocol would include T2weighted sequences (usually fast spin-echo sequences with respiratory gating) are optimal for evaluating soft tissue abnormalities involving the wall of the gall bladder [19]. The section thickness should be less than or equal to $5 \mathrm{~mm}$ with $1-2 \mathrm{~mm}$ gap. In addition, heavily T2-weighted fluid-sensitive acquisition techniques such as half-Fourier acquisition single-shot turbo spin echo (HASTE) can be utilized. Dynamic contrast materialenhanced fat-suppressed T1-weighted MRI sequences improve the delineation of the gall bladder wall and bile ducts and allow assessment of the liver parenchyma for

Table 5 Sensitivity and specificity of MDCT in detection of carcinoma of gall bladder

\begin{tabular}{|c|c|c|c|c|c|c|c|c|c|}
\hline $\begin{array}{l}\mathrm{CT} \\
\text { sensitivity }\end{array}$ & $\begin{array}{l}\text { CT } \\
\text { specificity }\end{array}$ & $\begin{array}{l}\text { MRI sensitivity } \\
\text { (liver invasion) }\end{array}$ & $\begin{array}{l}\text { MRI specificity } \\
\text { (liver invasion) }\end{array}$ & $\begin{array}{l}\text { MRI sensitivity } \\
\text { (biliary invasion/LNM) }\end{array}$ & $\begin{array}{l}\text { MRI specificity } \\
\text { (liver invasion/LNM) }\end{array}$ & $\begin{array}{l}\text { USG } \\
\text { sensitivity }\end{array}$ & $\begin{array}{l}\text { USG } \\
\text { specificity }\end{array}$ & $\begin{array}{l}\text { FDG PET-CT } \\
\text { sensitivity }\end{array}$ & $\begin{array}{l}\text { FDG } \\
\text { PET-CT sp }\end{array}$ \\
\hline $87.7 \%$ & $78.8 \%$ & $67 \%$ & $84 \%$ & $80.7 \%$ & $85 \%$ & $61 \%$ & $80 \%$ & $78 \%$ & $87 \%$ \\
\hline
\end{tabular}


tumor invasion and metastasis [20]. There is an emerging role of FDG PET (positron emission tomography) $\mathrm{CT}$ in diagnosis and staging of gall bladder neoplasms. This is due to its ability to combine anatomic and functional images for detection of lesions, lymph node involvement, and metastasis. However, its sensitivity is limited particularly for small volume peritoneal disease. The addition of PET to standard staging, CT may be helpful in $17 \%$ of patients to improve classification of doubtful lesions by CT or MRI and identify distant metastatic disease but may not be beneficial in 3\% of patients. FDG-PET seems to be complimentary rather than definitive in many patients with GBC according to a study.

A rare case of mucin producing carcinoma of gall bladder was described by few authors. This has interesting imaging findings as it presents as a cauliflower-like growth with small calcified foci in the gall bladder wall and laminated high viscosity fluid inside the gall bladder cavity. Dilatation of the cystic duct is also an associated feature. This type is believed to be more aggressive than adenocarcinoma [21].

A study conducted in one of the endemic regions highlighted few Unusual Imaging Features of Carcinoma Gall Bladder in a Tertiary Care Center [22]. The authors stated that hepatocellular carcinoma involving the gall bladder fossa can mimic GB malignancy. In their study, four cases presented with perforation and necrosis in the gall bladder fossa.

A case report published in South Asian Journal of Cancer in 2019 discussed metachronous muscle metastasis in a case of GBC with TP53 gene mutation. Another peculiar thing was the exceptional good outcome in the above patient as TP53 mutation is associated with very poor prognosis [23].

There are newer modalities with emerging role in imaging of GB pathologies. Contrast-enhanced ultrasound (CEUS) is a new modality which has applications in gall bladder pathology imaging like other organs like the liver, pancreas, and kidney. It shows vascularization in two phases-arterial (10-20 s after injection) and late phases (31-180 s). Gall bladder, unlike liver, has a single blood supply that is via cystic artery. The advantage of CEUS is that it can be safely used in patients with renal insufficiency. According to a retrospective study performed in Germany on 37 patients, CEUS imaging results showed sensitivity and specificity of $100 \%$, a positive predictive value of $100 \%$ and a negative predictive value of $100 \%$ [23]. However, according to a meta-analysis and systematic review [24] conducted by Yuan et al. in 2018, the pooled sensitivity and specificity of CEUS in differentiating between benign and malignant GB lesions were 0.81 and 0.87 , respectively. According to them, when compared malignant gall bladder lesions with benign ones, the contrast arrival time and the time to peak enhancement were significantly shorter in the latter. Discontinuity of gall bladder wall and infiltration to the surrounding tissue during enhancement suggest high possibility of malignancy. Tortuous intralesional vessels and thickened gall bladder wall may increase the predictive value for GB neoplasm [24].

In a retrospective study, the absence of enhancement on CEUS was present in 16/16 patients with sludge and in $0 / 23$ patients with lesions of the gall bladder (sensitivity and specificity 100\%). Washout was within $60 \mathrm{~s}$ in 9 out of 9 gall bladder carcinomas and 2 out of 14 benign lesions (sensitivity 100\%; specificity $85 \%$ ). In conclusion, CEUS improves the diagnostic accuracy of ultrasound. In doubtful cases, CEUS is very accurate in biliary sludge diagnosis. An intralesional washout at $60 \mathrm{~s}$ is a pattern of malignancy that can point towards a correct diagnosis, but it is limited by the presence of false positive results, especially for smaller lesions [25].

Elastography is an additional tool to enhance the diagnostic accuracy of ultrasound in differentiation of benign from malignant polyps as size criteria alone is not reliable. A prospective study aimed to evaluate the utility of real time elastography for gall bladder polyps and to demonstrate the elasticity properties of the polyps. In their study, all benign GB polyps showed high strain elastography pattern [26]. In another recent study, authors were able to evaluate point shear-wave elastography in the assessment of gall bladder polyps by collecting this diagnostic data and indicating the differences in the wave propagation between the two types of lesions: benign and malignant. Their findings confirm that all benign polyps have lower propagation velocities in comparison with the malignant ones. Their study showed that point shear-wave elastography (pSWE) of gall bladder lesions is feasible and can be useful for the differential diagnosis of GB neoplasm [27].

\section{Differentiating malignancy from other common pathologies Polyps}

Differentiation of a polyp from sludge is possible by changing the patient's position. Benign polyps include cholesterolosis (majority), adenomyosis, inflammatory, and hyperplastic types. As mentioned earlier (under pathogenesis), size and morphology constitute important criteria for deciding on management of suspicious polyps. The wall adjacent to the polyp should be normal, and irregularity or focal thickening $>3 \mathrm{~mm}$ can be a hint towards malignancy [12]. Age more than 40 years was also found to be a finding in resected polyps proved to be adenocarcinoma [13]. 
In a recent review, the authors concluded that although transabdominal ultrasound seems quite good at discriminating between gall bladder polyps and no polyps, it is less accurate in differentiating whether the polyp is a true or pseudo polyp and between dysplastic polyp/carcinoma or adenoma/pseudo polyp. In practice, this would lead to both unnecessary surgeries for pseudo polyps and missed cases of true polyps, dysplastic polyps, and carcinomas. There was insufficient evidence that endoscopic ultrasound is better compared to Transabdominal ultrasound in differentiating between true and pseudo polyps and between dysplastic polyps/carcinomas and adenomas/pseudo polyps [28].

\section{Adenomyomatosis}

It is characterized by cholesterol trapped within dilated Rokitansky-Aschoff sinuses which are represented by $\mathrm{V}$-shape comet tail artifacts on ultrasound. The most commonly described feature of adenomyomatosis on MR imaging is the "pearl necklace" sign, which results from the dilated Rokitansky-Aschoff sinuses [13]. It can be of focal and diffuse types with an enhancement pattern like that of GBC.

\section{Acute cholecystitis}

Gall bladder calculi are found more often in patients with acute cholecystitis and patients with GBC showed typical heterogeneous masses or focal enhanced wall thickening when compared to diffuse wall thickening in patients with acute cholecystitis [29] (Fig. 3). In a prospective study, the authors observed that acute cholecystitis most often showed a typical enhancement pattern, which consisted of a smooth weakly enhancing thin inner layer and a non-enhancing thick outer layer. The weakly enhancing thin inner layer is consistent with inflamed or sloughed mucosa, and the non-enhancing thick outer layer represents an edematous loose connective tissue layer [30].

\section{Xanthogranulomatous cholecystitis}

Xanthogranulomatous cholecystitis, an unusual variant of chronic cholecystitis, is characterized by focal or diffuse destructive inflammatory process, followed by fibrosis along with infiltration of macrophages and foamy cells. In a recent retrospective cohort study [16], it was shown on multivariate analysis that the following can be useful in making or excluding diagnosis of gall bladder cancer: focal gall bladder wall thickening ( $p=0.003)$, pericholecystic
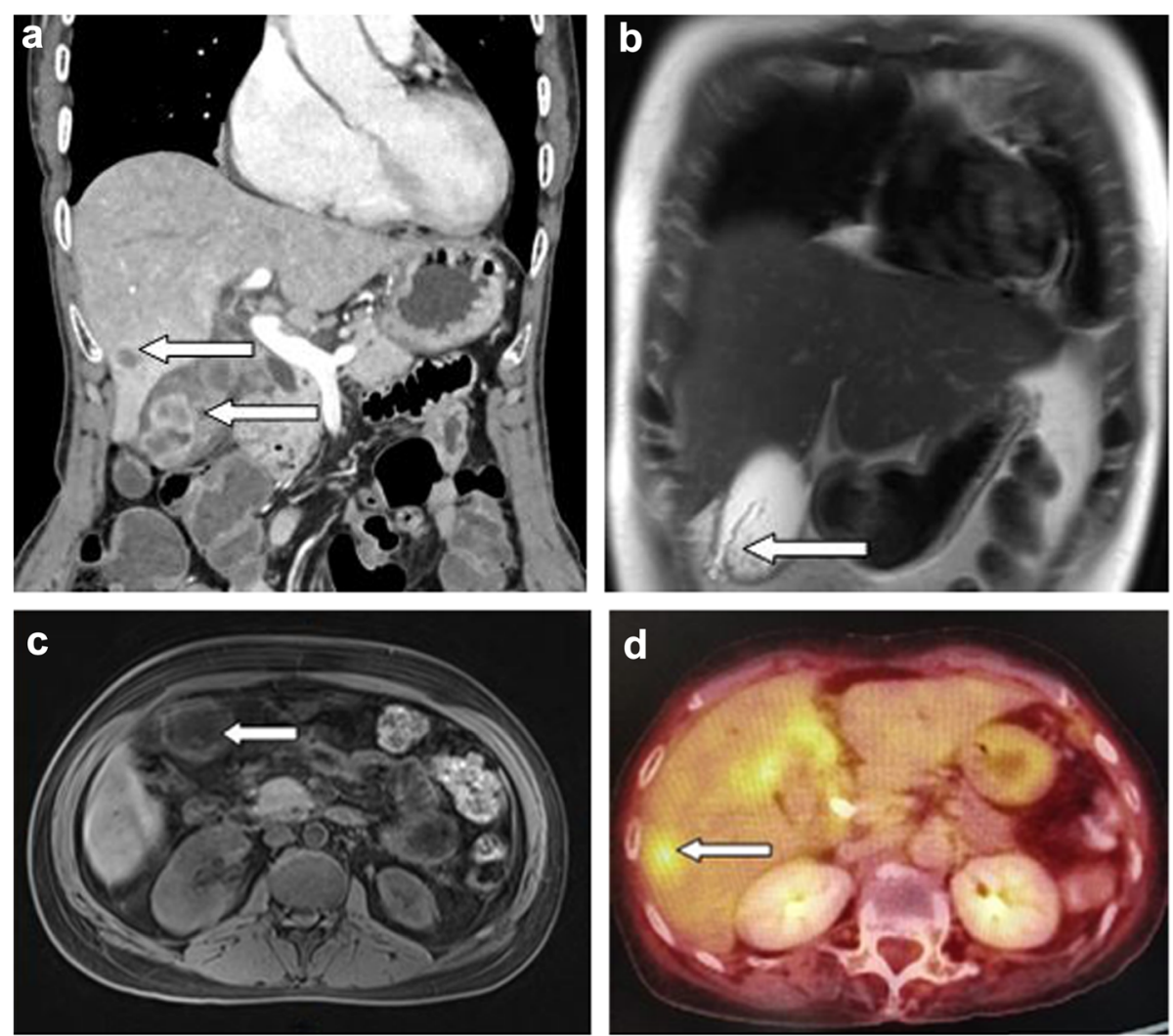

Fig. 3 (Clockwise) Coronal CECT image shows a heterogeneously enhancing advanced GBC with hepatic metastasis (a); coronal T2-weighted MR image and axial fat-suppressed image show acute cholecystitis mimicking GBC (b, c); FDG PET image shows increased uptake in the GB fossa as well as in the hepatic metastasis (d) 

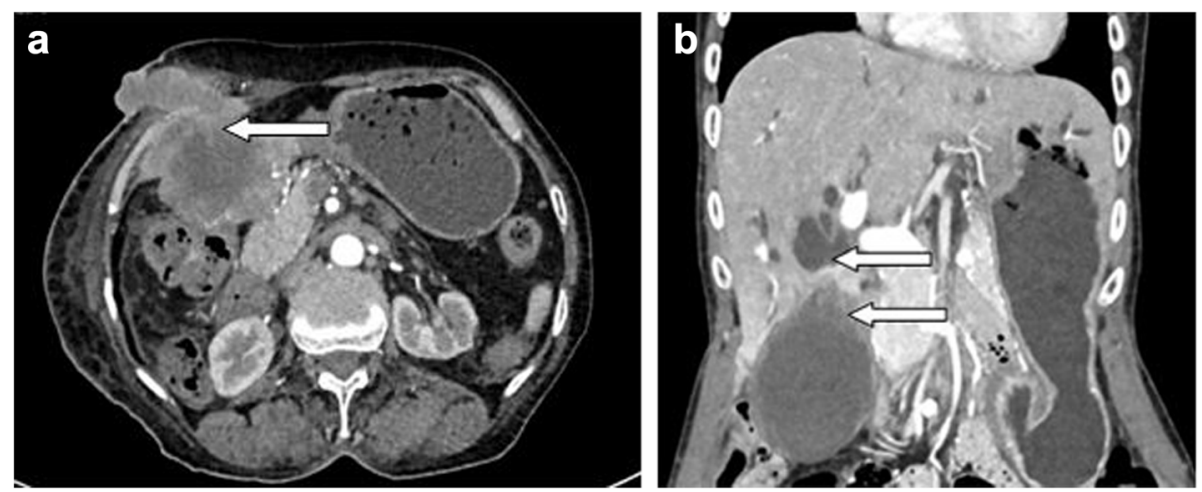

Fig. 4 Axial CECT image shows an irregular, exophytic mass lesion invading the anterior abdominal wall and the adjacent small bowel loops (a); coronal CECT image shows dilated intrahepatic biliary ducts due to an eccentric GB wall carcinoma (b)

"fat stranding" ( $p=0.018)$, and maximum short axis lymph node diameter $(p=0.043)$. The authors concluded that MDCT had moderate sensitivity and poor specificity for the differentiation of gall bladder cancer from acute cholecystitis and Xanthogranulomatous cholecystitis (XGC). Unlike other past studies, intramural hypo attenuating nodule, which has been described as a characteristic feature of Xanthogranulomatous cholecystitis, this study showed the same as having a poor predictive value in differentiation of XGC from malignancy.

In a mini review by authors from ILBS institute, New Delhi, India, salient differentiating features between XGC and gall bladder malignancy were described. A continuous mucosal lining is more often observed with XGC (66.7\% of cases) compared to a disrupted mucosal lining (33.3\%) as XGC is mainly pathology of gall bladder wall. XGC presenting as mass replacing gall bladder, intra-luminal mass or polypoidal mass-like thickening has not yet been described. Also, homogeneous enhancement of lymph nodes is more commonly seen in XGC than gall bladder malignancy. This study states that MR diffusion restriction was more frequently seen in GBC (68\%) than in XGC (7\%). The mean ADC value of XGC was higher than that of the wall-thickening type of gall bladder cancer with statistical significance [31].

\section{Conclusions}

The incidence of gall bladder carcinoma remains much higher in Indian than in the western world with a more delayed presentation thus carrying worse prognosis (Fig. 4). It is thus essential that it is recognized early and differentiated from other benign pathologies. Out of all available modalities, MDCT, FDG-PET CT, and image-guided biopsies play the most important role in diagnosis and follow-up. Imaging remains elementary in pre-operative planning and management of gall bladder neoplasms.

\section{Abbreviations}

GBC: Gall bladder carcinoma; AJCC: American Joint Committee on Cancer; U.P: Uttar Pradesh; CECT: Contrast-enhanced computed tomography; ICMR: Indian Council of Medical Research; FNAC: Fine needle aspiration cytology; WHO: World Health Organization; GB: Gall bladder; MDCT: Multidetector computed tomography; MRA: Magnetic resonance angiography; MRCP: Magnetic resonance cholangiopancreatography; PET: Positron emission tomography; ADC: Apparent diffusion coefficient; HASTE: Half-Fourier acquisition single-shot turbo spin echo; CEUS: Contrastenhanced ultrasound; pSWE: Point shear-wave elastography; XGC: Xanthogranulomatous cholecystitis

\section{Acknowledgements}

NA

\section{Authors' contributions}

Publication is approved by all authors and tacitly or explicitly by the responsible authorities where the work was carried out. NP: design of the work; analysis, interpretation of data; drafted the work and major contributor in writing the manuscript. SS design of the work; analysis; revised the work. Authors have approved the submitted version and have agreed both to be personally accountable for the authors' own contributions and to ensure that questions related to the accuracy or integrity of any part of the work, even ones in which the author was not personally involved, are appropriately investigated, resolved, and the resolution documented in the literature. All authors have read and approved the final manuscript.

\section{Funding}

None

Availability of data and materials

The datasets generated and/or analyzed during the current study are available in PubMed.

Ethics approval and consent to participate NA

\section{Consent for publication} NA

\section{Competing interests}

The authors declare that they have no competing interests.

\section{Author details}

'Department of Radiology, Dr.D.Y.Patil Medical College, Hospital \& Research Centre, Room No. A-42, Old Staff Quarter, Dr.D.Y.Patil Hospital, Pimpri, Pune, Maharashtra 411018, India. ${ }^{2}$ All India Institute of Medical Sciences, Patna, Bihar, India. ${ }^{3}$ Department of Radiology, Tata Medical Center, Kolkata, India. 
Received: 21 September 2020 Accepted: 13 December 2020

Published online: 05 January 2021

\section{References}

1. Furlan A, Ferris JV, Hosseinzadeh K, Borhani AA (2008) Gall bladder carcinoma update: multimodality imaging evaluation, staging, and treatment options. Am J Roentgenol 191(5):1440-1447

2. Dwivedi AN, Jain S, Dixit R (2015) Gall bladder carcinoma: aggressive malignancy with protean loco-regional and distant spread. World J Clin Cases 3(3):231

3. Kanthan R, Senger JL, Ahmed S, Kanthan SC (2015) Gall bladder cancer in the 21st century Journal of oncology

4. Jindal G, Singal S, Na B, Mittal A, Mittal S, Singal R (2018) Role of multidetector computed tomography (MDCT) in evaluation of gall bladder malignancy and its pathological correlation in an indian rural center. Maedica 13(1):55

5. Søreide K, Guest RV, Harrison EM, Kendall TJ, Garden OJ, Wigmore SJ (2019) Systematic review of management of incidental gall bladder cancer after cholecystectomy. Br J Surg 106(1):32-45

6. Patkar S, Shinde RS, Kurunkar SR, Niyogi D, Shetty NS, Ramadwar M, Goel M (2017) Radiological diagnosis alone risks overtreatment of benign disease in suspected gall bladder cancer: a word of caution in an era of radical surgery. Indian J Cancer 54(4):681

7. Hari Shankar et al (2019) Indian Council of Medical Research consensus document for the management of gall bladder cancer. Indian J Med Paediatric Oncol;36,2:79-84

8. Dixit R, Shukla VK (2014) Why is gall bladder cancer common in the gangetic belt? in perspectives in cancer prevention-translational cancer Research (pp. 145-151). Springer, New Delhi.

9. Jha V, Sharma P, Mandal KA (2018) Incidental gall bladder carcinoma: utility of histopathological evaluation of routine cholecystectomy specimens. South Asian J Cancer 7(1):21

10. Yadav R, Jain D, Mathur SR, Sharma A, lyer VK(2013) Gall bladder carcinoma: an attempt of WHO histological classification on fine needle aspiration material. Cytojournal 18; 10:12.

11. Maldonado CZ, Lopez MJ, Valverde FM, Soldevilla FA, Quirante FP, Medina VG(2014) Ultrasound findings associated to gall bladder carcinoma. Cirugía Española (English Edition). 1;92(5):348-355.

12. McKnight T, Patel A (2012) Gall bladder masses: multimodality approach to differential diagnosis. J Am Osteopathic Coll Radiol.9; 1(4):22-31.

13. Li Y, Tejirian T, Collins JC (2018) Gall bladder polyps: real or imagined?. Am Surg. 1;84(10):1670-1674.

14. Ozan E, Atac GK, Gundogdu S (2016) Twinkling artifact on color Doppler ultrasound: an advantage or a pitfall? J Med Ultrason 43(3):361-371

15. Mitchell CH, Johnson PT, Fishman EK, Hruban RH, Raman SP (2014) Features suggestive of gall bladder malignancy: analysis of T1, T2, and T3 tumors on cross-sectional imaging. J Comput Assist Tomography 38(2):235

16. Mathur M, Singh J, Singh DP, Kaur N, Gupta S, Haq S (2017) Imaging evaluation of enhancement patterns of flat gall bladder wall thickening and its correlation with clinical and histopathological findings. J Clin Diagn Res 11(4):TCO7

17. Jung SE, Lee JM, Lee K, Rha SE, Choi BG, Kim EK, Hahn ST(2005) Gall bladder wall thickening: MR imaging and pathologic correlation with emphasis on layered pattern. Eur Radiol. 1;15(4):694-701.

18. Prasad N, Goyal A (2018) MDCT in characterizing gall bladder carcinoma: experience from an endemic region. Int J Contemp Med Surg Radiol 3(2): B123-B126

19. Tan CH, Lim KS (2013) MRI of gall bladder cancer. Diagn Interv Radiol 19(4):312

20. Leung U, Pandit-Taskar N, Corvera CU, D'Angelica MI, Allen PJ, Kingham TP, DeMatteo RP, Jarnagin WR, Fong Y (2014) Impact of pre-operative positron emission tomography in gall bladder cancer. HPB. 16(11):1023-1030

21. Huang CP, Chiou YY, Chou YH, Chiang JH, Chang CY (2006) Imaging findings in mucin-producing carcinoma of the gall bladder. J Formos Med Assoc. 1;105(5):427-430

22. Mehra R, Das RK, Singh GN (2017) Unusual imaging features in carcinoma gall bladder in a tertiary referral Centre. Paripex Indian J Res 6:45-47

23. Ghosh J, Ganguly S, Dabkara D, Biswas B, Chatterjee A, Mukhopadhyay S, Chandra A, Sen S, Dey D (2019) Metachronous muscle metastasis in a case of metastatic gall bladder cancer with TP35 gene mutation: a rare case report. South Asian J Cancer 8(4):240
24. Negrão de Figueiredo G, Mueller-Peltzer K, Armbruster M, Rübenthaler J, Clevert DA (2019) Contrast-enhanced ultrasound (CEUS) for the evaluation of gall bladder diseases in comparison to cross-sectional imaging modalities and histopathological results. Clin Hemorheol Microcirc 71(2):141-149

25. Cheng Y, Manni Wang BM, Ma X (2018) Potential role of contrast-enhanced ultrasound for the differentiation of malignant and benign gall bladder lesions in East Asia: a meta-analysis and systematic review. Medicine. 97(33)

26. Serra C, Felicani C, Mazzotta E, Gabusi V, Grasso V, De Cinque A, Giannitrapani L, Soresi M (2018) CEUS in the differential diagnosis between biliary sludge, benign lesions and malignant lesions. J Ultrasound 21(2):119-126

27. Teber MA, Tan S, Dönmez U, Ipek A, Uçar AE, Yıldırım H, Aslan A, Arslan H (2014) The use of real-time elastography in the assessment of gall bladder polyps: preliminary observations. Med Ultrasonography 16(4):304-308

28. Doykov D, Andonov V (2019) Assessment of gall bladder polyps using point shear-wave elastography: a preliminary observation. J Sci Res Studies 6(2):11-14

29. Wennmacker SZ, Lamberts MP, Di Martino M, Drenth JP, Gurusamy KS, van Laarhoven CJ (2018) Transabdominal ultrasound and endoscopic ultrasound for diagnosis of gall bladder polyps. Cochrane Database Syst Rev 8

30. Kim SH, Jung D, Ahn JH, Kim KS (2017) Differentiation between gall bladder cancer with acute cholecystitis: considerations for surgeons during emergency cholecystectomy, a cohort study. Int J Surg 45:1-7

31. Wasnik AP, Davenport MS, Kaza RK, Weadock WJ, Udager A, Keshavarzi N, Nan B, Maturen KE (2018) Diagnostic accuracy of MDCT in differentiating gall bladder cancer from acute and xanthogranulomatous cholecystitis. Clin Imaging 50:223-228

\section{Publisher's Note}

Springer Nature remains neutral with regard to jurisdictional claims in published maps and institutional affiliations.

\section{Submit your manuscript to a SpringerOpen ${ }^{\circ}$ journal and benefit from:}

- Convenient online submission

- Rigorous peer review

- Open access: articles freely available online

High visibility within the field

- Retaining the copyright to your article

Submit your next manuscript at $\boldsymbol{\nabla}$ springeropen.com 